Applied Mathematical Sciences Vol. 8, 2014, no. 134, 6745 - 6752

HIKARI Ltd, www.m-hikari.com

http://dx.doi.org/10.12988/ams.2014.49689

\title{
A Survey on Sylow Normalizers and Classes of Groups
}

\author{
A. D’Aniello( ${ }^{(1)}$, L. S. Kazarin ${ }^{(2)}$, A. Martínez-Pastor ${ }^{(3)}$ \\ and M. D. Pérez-Ramos ${ }^{(4)}$ \\ (1) Dipartimento di Matematica e Applicazioni "R. Caccioppoli" \\ Università di Napoli "Federico II" - via Claudio 21, 80125 Napoli, Italy \\ ${ }^{(2)}$ Department of Mathematics, Yaroslavl P. Demidov State University \\ Sovetskaya Str 14, 150000 Yaroslavl, Russia \\ ${ }^{(3)}$ Instituto Universitario de Matemática Pura y Aplicada IUMPA \\ Universidad Politécnica de Valencia \\ Camino de Vera, s/n, 46022 Valencia, Spain \\ ${ }^{(4)}$ Departament d'Àlgebra, Universitat de València \\ C/ Doctor Moliner 50, 46100 Burjassot (València), Spain
}

Copyright (c) 2014 A. D'Aniello et al. This is an open access article distributed under the Creative Commons Attribution License, which permits unrestricted use, distribution, and reproduction in any medium, provided the original work is properly cited.

\begin{abstract}
We report on a stream of research in relation with Sylow normalizers, i.e. normalizers of Sylow subgroups, of finite groups and group's classes.
\end{abstract}

Mathematics Subject Classification: 20D20, 20F17, 20D15

Keywords: Finite groups; Sylow normalizers; saturated formations; nilpotent Hall Subgroups

\section{From local to global}

Sylow theorems may be seen as a first sign of the great influence that local theory has played in the study of finite groups. This theory deals with the 
analysis of the structure of finite groups in relation to a fixed prime $p$, and rests on the fact that local properties, such as those of $p$-subgroups and their normalizers, influence the global structure of the groups. Local finite group theory was initially driven to settle the solubility of groups of odd order within the classification of finite simple groups. All groups considered in this note are finite. We aim to report about a more recent stream of research arising from the following question in the spirit of local theory: which properties of the Sylow normalizers, i.e., normalizers of Sylow subgroups, are inherited by the whole group?

A classical result of Glauberman [13] states that a group is a $p$-group for a prime $p$ if and only if its Sylow subgroups are self-normalizing. In [11] this result is read off as: if $\mathcal{H}=\bigcup_{p \in \mathbb{P}} \mathcal{S}_{p}, \mathbb{P}$ the set of all primes and $\mathcal{S}_{p}$ the class of all $p$-groups, then a group $G$ belongs to the class $\mathcal{H}$ if and only if its Sylow normalizers belong to $\mathcal{H}$. Bianchi, Gillio Berta Mauri and Hauck [4] proved that an analogous result remains true if the class $\mathcal{H}$ is replaced by the class of all nilpotent groups. Fedri and Serena [11] pointed out that there are non abelian simple groups with supersoluble Sylow normalizers; also the symmetric group of degree 4 is a soluble non-supersoluble group whose Sylow normalizers are supersoluble. In [11], continued in [5] in collaboration with Bryce, the structure of groups with supersoluble Sylow normalizers was investigated. But it is still true that a group is nilpotent whenever the normalizer of each Sylow $p$ subgroup is $p$-nilpotent for every prime $p$, as proved by Ballester-Bolinches and Shemetkov [2]. We emphasize at this point that classes of groups, being collections of groups closed under isomorphisms, provide a convenient framework for this investigation. D'Aniello, De Vivo and Giordano [6, 7] led the study towards the theory of classes of groups and focused on closure properties of the class

$$
\mathrm{N} \mathcal{X}=\left(G \mid N_{G}(P) \in \mathcal{X} \forall P \in \operatorname{Syl}(G)\right)
$$

in relation with the closure properties of a given class $\mathcal{X}$, where $\operatorname{Syl}(G)$ denotes the set of all Sylow subgroups of the group $G$. In the first reference these authors proved that a group is primitive if the Sylow normalizers are primitive. In [7] conditions are found when some classes of soluble groups, defined by properties of the Sylow normalizers, are saturated formations. In particular, the class of soluble groups whose normalizers of Sylow $p$-subgroups are $p$-supersoluble, for a given prime $p$, is a saturated formation.

The theory of saturated formations keeps the flavour of local theory with the celebrated theorem of Gaschütz-Lubeseder-Schmid which states that nonempty saturated formations are exactly local formations. We refer to the book of Doerk and Hawkes [10] for convenient background about classes of groups and theories of formations and Fitting classes. The class of nilpotent groups constitutes the most elementary model for saturated formations, if one thinks of nilpotent groups as groups whose chief factors are central. This is to say 
that the automorphism group $\operatorname{Aut}_{G}(\mathrm{C})=G / C_{G}(\mathrm{C})$, that the group $G$ induces on each $p$-chief factor $\mathrm{C}=H / K$ of $G$ for every prime $p$, belongs to the trivial formation $f(p)=(1)$. (Here the trivial formation may be equivalently replaced by the formation $f(p)=\mathcal{S}_{p}$ of all $p$-groups.) A $p$-chief factor of a group is a chief factor whose order is divisible by the prime $p$. We recall next some basic concepts on formations.

$A$ formation $\mathcal{F}$ is a class of groups such that $G / N \in \mathcal{F}$ if $G \in \mathcal{F}$ and $N \unlhd G$, and if $G / N_{1} \cap N_{2} \in \mathcal{F}$ whenever $G / N_{1}, G / N_{2} \in \mathcal{F}$. A formation $\mathcal{F}$ is said to be saturated if $G \in \mathcal{F}$ whenever $G / \Phi(G) \in \mathcal{F}$.

$A$ function $f$ which associates a formation $f(p)$ to each prime $p \in \mathbb{P}$ is called a formation function. Then the class

$$
L F(f):=\left(G \mid \operatorname{Aut}_{G}(\mathrm{C}) \in f(p) \text { for each } p \text {-chief factor } \mathrm{C}, \forall p \in \mathbb{P}\right)
$$

is a formation which is said to be locally defined by the formation function $f$.

A class of groups $\mathcal{F}$ is called a local formation if there exists a formation function $f$ such that $\mathcal{F}=L F(f)$. It is useful to note that $\mathcal{F}=L F(f)=$ $\mathcal{E}_{\pi} \cap\left(\cap_{p \in \pi} \mathcal{E}_{p^{\prime}} \mathcal{E}_{p} f(p)\right)$, where $\pi=\operatorname{Char}(\mathcal{F})=\{p \in \mathbb{P} \mid f(p) \neq \emptyset\}$ is the characteristic of $\mathcal{F}$ and, for any set of primes $\sigma, \mathcal{E}_{\sigma}$ is the class of all $\sigma$-groups.

\section{Nilpotent-like Fitting formations}

In the literature the term nilpotent-like Fitting formation refers to a wide variety of classes of groups which appear initially as generalization of the class of nilpotent groups. They are Fitting classes and saturated formations, locally defined by a formation function $f$ given by $f(p)=\mathcal{E}_{\pi(p)}$, where either $\pi(p)$ is a non-empty set of primes containing the prime $p$ or $\pi(p)=\emptyset$, for each prime $p \in \mathbb{P}$.

In a number of papers nilpotent-like Fitting formations have been shown to share some important aspects with the class of nilpotent groups: They describe as local formations those subgroup-closed saturated formations such that every critical group is either a Schmidt group or a cyclic group of prime order, which are also characterized by the validity of an extension of the Frobenius $p$-nilpotence criterion. Recall that with respect to a class of groups $\mathcal{X} a$ group $G$ is critical if $G$ is not in $\mathcal{X}$ but all proper subgroups of $G$ are in $\mathcal{X}$ and that Schmidt groups are the critical groups for the class $\mathcal{N}$ of all nilpotent groups. Moreover, various restrictions on the sets of primes $\pi(p)$, locally defining the formation, ensure extensions of the class of nilpotent groups from different points of views and in a variety of frameworks. In this direction relevant results appear in relation with subgroup lattices, factorized groups, formations and Fitting classes (see [1] for an account of this development). A further insight appears in $[9,16,17]$ in relation with Sylow normalizers. Again 
a convenient restriction on the sets of primes locally defining a nilpotent-like Fitting formation leads to the so-called covering formations, which are classes of groups with nilpotent Hall subgroups for adequate sets of primes and play an important role in relation with Sylow normalizers (see Section 4). More information about these topics can be found in the book of Guo [14, Chapter 3].

Finally, a study in [12] deals with the influence of 2-generator subgroups on the structure of groups and factorized groups. Here nilpotent-like Fitting formations of soluble groups are characterized like certain saturated formations $\mathcal{F}$ of soluble groups which are closed under taking products of $\mathcal{F}$-connected $\mathcal{F}$-subgroups. We recall that for a non-empty class of groups $\mathcal{C}$, two subgroups $A$ and $B$ are called $\mathcal{C}$-connected if $\langle a, b\rangle \in \mathcal{F}$ for all $a \in A$ and all $b \in B$.

In spite of the name and initial developments, the concept of nilpotent-like Fitting formation comprises interesting saturated formations that only vaguely resemble the class of nilpotent groups. For instance, given any sets $\pi$ and $\rho$ of primes, the class $\mathcal{E}_{\pi} \mathcal{E}_{\rho}$ of all groups that are extensions of $\pi$-groups by $\rho$-groups is a nilpotent-like Fitting formation as well as the class of all groups with a Sylow tower for any given total ordering of the primes. Many more examples are described in [12].

\section{The Sylow graph of a group}

A main goal in the course of this research is the connectedness of the so-called Sylow graph of a group [16, Main Theorem].

Let $G$ be a group and $G_{p}$ a Sylow p-subgroup of $G$ for any prime $p \in \pi(G)$, the set of prime divisors of the order of $G$. Then the automiser $A_{p}(G)$ is the group $N_{G}\left(G_{p}\right) / G_{p} C_{G}\left(G_{p}\right)$, up to conjugacy of Sylow subgroups. The Sylow graph $\Gamma_{A}(G)$ of the group $G$, with set of vertices $\pi(G)$, is defined as follows: Two vertices $p, q \in \pi(G)$ are joined if either $q \in \pi\left(A_{p}(G)\right)$ or $p \in \pi\left(A_{q}(G)\right)$.

Theorem 3.1. Let $G$ be a finite almost simple group. Then the graph $\Gamma_{A}(G)$ is connected and has diameter at most 5.

It follows from this theorem that often (with few exceptions) the normalizer of a Sylow $p$-subgroup $G_{p}$ of a finite almost simple group $G$ is larger than $G_{p} C_{G}\left(G_{p}\right)$, for $p$ an odd prime. In particular, this is always true if $G$ is a finite simple group (see [15]). More precise information is obtained in [16] in graph terms for almost simple groups. 


\section{Covering formations, Nilpotent Hall sub- groups and Sylow normalizers}

The definition of covering formation is motivated by the following basic fact in relation with Sylow normalizers. For two primes $p, q, p \neq q$, we denote $\left[V_{p}\right] Z_{q}$ the primitive $\{p, q\}$-group with stabilizer the cyclic group $Z_{q}$.

Proposition 4.1. [9, Proposition 1] Let $\mathcal{F}$ be a subgroup-closed saturated formation such that $\mathrm{N} \mathcal{F} \cap \mathcal{S} \subseteq \mathcal{F}$, and let $p, q \in \operatorname{Char}(\mathcal{F})$. Then $\left[V_{p}\right] Z_{q} \in \mathcal{F}$ implies that $\left[V_{q}\right] Z_{p} \in \mathcal{F}$.

Next we present the main concepts of this section.

Let $\pi$ be a set of primes. For each prime $p \in \pi$, let $\pi(p)$ be a set of primes satisfying the following conditions:

(i) $p \in \pi(p) \subseteq \pi$,

(ii) for any $q \in \pi(p)$, then $p \in \pi(q)$. (Write $p \leftrightarrow q$, in this case.)

Set $\Sigma:=\{\sigma \subseteq \pi|| \sigma \mid \geq 2,(p, q \in \sigma, p \neq q \Rightarrow p \not \rightarrow q)\}$.

Associated to such sets of primes, we consider the following classes of groups:

- $\mathcal{F}=L F(f) \quad$ with $f(p)=\mathcal{E}_{\pi(p)}$ if $p \in \pi, \quad f(q)=\emptyset$ if $q \notin \pi$.

Such nilpotent-like Fitting formations $\mathcal{F}$ appear in [9] where are called covering-formations.

- $\mathcal{U}$ denotes the class of all groups with nilpotent Hall $\sigma$-subgroups for all $\sigma \in \Sigma$, equivalently for all $\sigma \in \Sigma$ with $|\sigma|=2$ by [18, Lemma 3.4].

- $\mathcal{V}$ denotes the class of all groups whose $\sigma$-subgroups are nilpotent for all $\sigma \in \Sigma$, equivalently for all $\sigma \in \Sigma$ with $|\sigma|=2$ (easily deduced from the structure of Schmidt groups).

(In case $\Sigma=\emptyset$, it is understood $\mathcal{U}=\mathcal{V}=\mathcal{E}$.)

It is proved in [17, Proposition 2.1] that $\mathcal{F} \subseteq \mathcal{U} \subseteq \mathcal{V}$.

Recent characterizations of groups having nilpotent Hall subgroups, in terms of either Sylow numbers or class sizes, and related results appear in $[18,3]$.

A particular construction is given in the next example. In the soluble universe, the classes in Example 4.2 have been called lattice formations in [1], since they are characterized in terms of lattice properties of $\mathcal{F}$-subnormal subgroups.

Example 4.2. Assume that the sets of primes $\pi(p)$ form a partition $\left\{\pi_{i} \mid\right.$ $i \in I\}$ of $\pi$, then the groups in $\mathcal{F}$ are characterized by being direct products of Hall subgroups corresponding to the sets of primes in the partition, that is,

$$
\mathcal{F}=\times_{i \in I} \mathcal{E}_{\pi_{i}}:=\left(G \in \mathcal{E}_{\pi} \mid G=\times_{i \in I} G_{\pi_{i}}, G_{\pi_{i}} \in \mathcal{E}_{\pi_{i}}\right)
$$


We report next about main results in relation with covering formations and Sylow normalizers, first in the universe of soluble groups $\mathcal{S}$, and then in the universe of all finite groups $\mathcal{E}$.

\section{The soluble universe $\mathcal{S}$}

Theorem 4.3. [9, Proposition 2, Theorem] In the universe of soluble groups, covering formations coincide with the classes of groups with nilpotent Hall subgroups for adequate sets of primes, more precisely, $\mathcal{F} \cap \mathcal{S}=\mathcal{U} \cap \mathcal{S}=\mathcal{V} \cap \mathcal{S}$.

In addition, these classes characterize those subgroup-closed saturated formations $\mathcal{X} \subseteq \mathcal{S}$ such that a soluble group belongs to $\mathcal{X}$ if and only if its Sylow normalizers do also so.

See also [14, Theorems 3.5.14, 3.6.9].

\section{The finite universe $\mathcal{E}$}

The result on the Sylow graph is applied in [16] to prove the following:

Theorem 4.4. Let $\mathcal{F}=\times_{i \in I} \mathcal{E}_{\pi_{i}}$ with $\pi_{i} \cap \pi_{j}=\emptyset$ for $i \neq j$, as in Example 4.2. Then $G \in \mathcal{F}$ if and only if $N_{G}(P) \in \mathcal{F}$ for all $P \in \operatorname{Syl}(G)$.

In addition, with the previous notation, $\mathcal{F}=\mathcal{U}=\mathcal{V}$.

Similar results for other particular examples appear in [16].

However, we emphasize the following facts in the finite universe $\mathcal{E}$ :

- Examples in [17] show that $\mathcal{F} \neq \mathcal{U} \neq \mathcal{V}, \mathcal{F}=\mathcal{U} \neq \mathcal{V}$ and $\mathcal{F} \neq \mathcal{U}=\mathcal{V}$ are possible.

$\mathcal{U}$ is a formation but not subgroup-closed and not saturated in general. $\mathcal{U}$ is saturated if and only if $\mathcal{U}=\mathcal{F}$. (See [17, Theorem 2.2] for detailed conditions on $\mathcal{U}$ to insure this property.)

$\mathcal{V}$ is a subgroup-closed formation but not saturated in general. $\mathcal{V}$ is saturated if and only if $\mathcal{V}=\mathcal{F}$. (See [17, Theorem 2.1] for detailed conditions on $\mathcal{V}$ to insure this property.)

$[17$, Theorem 3.3] $\mathcal{F}=\mathcal{U}=\mathcal{V}$ if and only if every critical group with respect to $\mathcal{F}$ is either a Schmidt group or a cyclic group of prime order.

$[17$, Theorem 3.2] $\mathrm{N} \mathcal{F}=\mathcal{F}$ implies that $\mathcal{F}=\mathcal{U}=\mathcal{V}$.

- In general, $\mathrm{N} \mathcal{F} \neq \mathcal{F}$, as shown by the above-referred examples and the last mentioned result in [17, Theorem 3.2].

But also Examples 3.1 in [17] show that $\mathcal{F}=\mathcal{U}=\mathcal{V}$ is not enough to guarantee that $\mathrm{N} \mathcal{F}=\mathcal{F}$ (even if $\mathcal{F} \subseteq \mathcal{S}$ or with $\operatorname{Char}(\mathcal{F})=\mathbb{P}$ ). In particular, the class of groups all whose $\{2,3\}$-subgroups are nilpotent coincides with the class of groups with nilpotent Hall $\{2,3\}$-subgroups. This is a covering formation $\mathcal{F}$ which satisfies the equivalent conditions in [17, Theorem 3.3], i.e. every critical group is either a Schmidt group or a cyclic group of prime order, but $\mathrm{N} \mathcal{F} \neq \mathcal{F}$. 
- In general, a subgroup-closed saturated formation $\mathcal{X}$ such that $\mathrm{N} \mathcal{X}=\mathcal{X}$ is not a covering formation: [8, 3. Example] Let $p \in \mathbb{P} \backslash\{2,3,5,7,13\}$, $\pi=\{2,5,7,13, p\}$ and $\mathcal{H}=L F(h)$ given by $h(q)=\mathcal{E}_{\pi}$ if $q \in \pi \backslash\{p\}, h(p)=\mathcal{S}_{\pi}$, $h(t)=\emptyset$ if $t \notin \pi$. Then $\mathcal{H}$ is not a covering formation but $\mathrm{N} \mathcal{H}=\mathcal{H}$.

The class $\mathcal{H} \times \mathcal{E}_{\pi^{\prime}}=\left(G \mid G=A \times B, A \in \mathcal{H}, B \in \mathcal{E}_{\pi^{\prime}}\right)$ gives an example with full characteristic; see [16, Lemma 5.7].

Proposition 3.2 and Theorem 3.1 in [17] give conditions to guarantee that a subgroup-closed saturated formation $\mathcal{H}$ satisfying $\mathrm{N} \mathcal{H}=\mathcal{H}$ is a covering formation, in terms of the canonical local definition of $\mathcal{H}$.

Acknowledgements. The third and fourth authors have been supported by Proyecto MTM2010-19938-C03-02, Ministerio de Economía y Competitividad, Spain. The second author has been supported by RFBR Project 13-0100469.

\section{References}

[1] A. Ballester-Bolinches, A. Martínez-Pastor, M. C. Pedraza-Aguilera and M. D. Pérez-Ramos, On nilpotent-like Fitting formations, in: C. $M$. Campbell et al.(Eds.), Proceedings of Groups-St Andrews 2001 in Oxford, London Math. Soc. Lecture Note Ser. 304, Vol. 1, Cambridge University Press, 2003, 31 - 38.

[2] A. Ballester-Bolinches and L.A. Shemetkov, On Normalizers of Sylow Subgroups in Finite Groups, Siberian Math. J., 40 (1) (1999), 1 - 2.

[3] A. Beltrán, M.J. Felipe, G. Malle, A. Moretó, G. Navarro, L. Sanus, R. Solomon and P.H. Tiep, Nilpotent and abelian Hall subgroups in finite groups, to appear in Trans. Amer. Math. Soc.

[4] M. Bianchi, A. Gillio Berta Mauri and P. Hauck, On finite groups with nilpotent Sylow normalizers, Arch. Math., 47 (1986), 193 - 197.

[5] R.A. Bryce, V. Fedri and L. Serena, Bounds on the Fitting length of finite soluble groups with supersoluble Sylow normalizers, Bull. Austral. Math. Soc., 44 (1991), 19 - 31.

[6] A. D'Aniello, C. De Vivo and G. Giordano, Finite groups with primitive Sylow normalizers, Bolletino U.M.I., 8 5-B (2002), 235 - 245.

[7] A. D'Aniello, C. De Vivo and G. Giordano, Saturated formations and Sylow normalizers, Bull. Austral. Math. Soc., 69 (2004), 25 - 33.

[8] A. D'Aniello, C. De Vivo and G. Giordano, On certain saturated formations of finite groups, in: T. Hawkes, P. Longobardi, M. Maj (Eds.) 
Proceedings Ischia Group Theory 2006, World Scientific, River Edge, NJ, 2006, $22-32$.

[9] A. D’Aniello, C. De Vivo, G. Giordano and M.D. Pérez-Ramos, Saturated formations closed under Sylow normalizers, Comm. Algebra, 33 (2005), $2801-2808$.

[10] K. Doerk and T. Hawkes, Finite soluble groups, Walter De Gruyter, Berlin-New York, 1992.

[11] V. Fedri and L. Serena, Finite soluble groups with supersoluble Sylow normalizers, Arch. Math., 50 (1988), 11 - 18.

[12] M.P. Gállego, P. Hauck and M.D. Pérez-Ramos, Saturated formations and products of connected subgroups, J. Algebra, 333 (2011), 105 - 119.

[13] G. Glauberman, Prime-power factor groups of finite groups II, Math. Z., 117 (1970), 46 - 56.

[14] W. Guo, The theory of classes of groups, Mathematics and its Applications 505, Kluwer Academic Publishers Group, Dordrecht; Science Press, Beijing, 2000.

[15] R.M. Guralnick, G. Malle and G. Navarro, Self-normalizing Sylow subgroups, Proc. Amer. Math. Soc., 132 (2003), 273 - 279

[16] L. Kazarin, A. Martínez-Pastor and M.D. Pérez-Ramos, On the Sylow graph of a group and Sylow normalizers, Israel J. Math., 186 (2011), $251-271$.

[17] L. Kazarin, A. Martínez-Pastor and M.D. Pérez-Ramos, On Sylow normalizers of finite groups, J. Algebra Appl., 13(3) (2014), 1350116 - 1 20 .

[18] A. Moretó, Sylow numbers and nilpotent Hall subgroups, J. Algebra, 379 (2013), $80-84$.

\section{Received: May 21, 2014}

